\title{
PRO-DEFINABILITY OF SPACES OF DEFINABLE TYPES
}

\author{
PABLO CUBIDES KOVACSICS AND JINHE YE
}

(Communicated by Heike Mildenberger)

\begin{abstract}
We show pro-definability of spaces of definable types in various classical complete first order theories, including complete o-minimal theories, Presburger arithmetic, $p$-adically closed fields, real closed and algebraically closed valued fields and closed ordered differential fields. Furthermore, we prove pro-definability of other distinguished subspaces, some of which have an interesting geometric interpretation.

Our general strategy consists of showing that definable types are uniformly definable, a property which implies pro-definability using an argument due to E. Hrushovski and F. Loeser. Uniform definability of definable types is finally achieved by studying classes of stably embedded pairs.
\end{abstract}

\section{INTRODUCTION}

In [13, building on the model theory of algebraically closed valued fields (ACVF), E. Hrushovski and F. Loeser developed a theory which provides a model-theoretic account of the Berkovich analytification of algebraic varieties. Most notably, they obtained results concerning the homotopy type of quasi-projective varieties which were only known under strong algebro-geometric hypothesis on $X$ by results in [1].

One of the difficulties to study Berkovich spaces from a model-theoretic point of view is that such spaces do not seem to generally have (in ACVF) the structure of a definable set -where usual model-theoretic techniques can be applied- but rather canonically the structure of a space of types. Part of the novelty of HrushovskiLoeser's work lies on the fact that their spaces can be equipped with the structure of a (strict) pro-definable set, which granted them back the use of different classical model-theoretic tools. It is thus tempting to ask if such a structural result holds for other distinguished subsets of definable types and even for other first-order theories. It turned out this question is closely related to classical topics in model theory such as the model theory of pairs and uniform definability of types. In this article we give a positive answer in various contexts. Formally, we obtain the following result

Main Theorem 1 (Theorem 6.4). Let $T$ be one of the following theories: a complete o-minimal theory; Presburger arithmetic; the theory of a finite extension of

Received by the editors June 3, 2020, and, in revised form, November 23, 2020, and February 8, 2021.

2020 Mathematics Subject Classification. Primary 11S80; Secondary 11U09.

Key words and phrases. Pro-definability, definable types, stably embedded pairs.

The first author was partially supported by the ERC project TOSSIBERG (Grant Agreement 637027), ERC project MOTMELSUM (Grant agreement 615722) and individual research grant Archimedische und nicht-archimedische Stratifizierungen höherer Ordnung, funded by the DFG. The second author was partially supported by NSF research grant DMS1500671.

(C) 2021 by the authors under Creative Commons Attribution-Noncommercial 3.0 License (CC BY NC 3.0) 
$\mathbb{Q}_{p}$; the theory of real closed valued fields RCVF; a completion of the theory of algebraically closed valued fields ACVF; the theory of closed ordered differential fields CODF. Then, definable types over models of $T$ are uniformly definable. In particular, for every model $M$ of $T$ and every $M$-definable set $X$, the space $S_{X}^{\operatorname{def}}(M)$ of $M$-definable types concentrating on $X$ is pro-definable in $\mathcal{L}^{\mathrm{eq}}$.

The fact that pro-definability follows from uniform definability of types goes back to an argument of E. Hrushovski and F. Loeser in 13 which we present in Proposition 4.1. In return, uniform definability of types is obtained from the following criterion which relates it to stably embedded pairs of models of $T$.

Main Theorem 2 (Corollary 6.2). Let $T$ be an $\mathcal{L}$-theory such that

( $i)$ the class of stably embedded pairs of models of $T$ is elementary in the language of pairs;

(ii) for every small model $M \models T$ and every definable type $p \in S_{x}^{\mathrm{def}}(M)$, there is a stably embedded pair $(N, M)$ such that $p$ is realized in $N$.

Then $T$ has uniform definability of types.

Ensuring point $(i)$ for the above-listed theories makes use of characterizations of definable types à la Marker-Steinhorn. In the case of $\mathrm{RCVF}$ and $p \mathrm{CF}_{e, f}$, we prove such a characterization (Theorem [5.9) in the spirit of [6].

As a corollary we also obtain pro-definability of some distinguished subspaces of the space of definable types which have a geometric interpretation. In particular, we aim to show that there are spaces of definable types in ACVF that can mimic Huber's analytification of an algebraic variety in a similar way the space of generically stable types mimics its Berkovich analytification. We hope this can serve as a basis towards a model theory of adic spaces. In the same spirit, working in RCVF, there are spaces of definable types which can be seen as the model-theoretic counterpart of the analytification of semi-algebraic sets as recently defined by P. Jell, C. Scheiderer and J. Yu in [14]. This article aims to lay down a foundation for a model-theoretic study of such spaces. In a sequel, we will further explore structural properties of some of these spaces.

A natural question to ask is if such spaces are also strict pro-definable (i.e., prodefinable with surjective transition maps). Obtaining strictness is much more subtle and is often related to completions of theories of stably embedded pairs. Results towards a positive answer to this question will also be addressed in subsequent work.

\section{Preliminaries AND NOtation}

2.1. Model theoretic background. Let $\mathcal{L}$ be a first order language (possibly multi-sorted) and $T$ be a complete $\mathcal{L}$-theory. The sorts of $\mathcal{L}$ are denoted by bold letters $\mathbf{D}$. Given a variable $x$, we let $\mathbf{D}_{x}$ denote the sort where $x$ ranges. If $M$ is a model of $T$ and $\mathbf{D}$ be a sort of $\mathcal{L}$, we let $\mathbf{D}(M)$ denote the set of elements of $M$ which are of sort $\mathbf{D}$. Given an ordered tuple of variables $x=\left(x_{i}\right)_{i \in I}$ (possibly infinite), we extend this notation and set

$$
\mathbf{D}_{x}(M)=\prod_{i \in I} \mathbf{D}_{x_{i}}(M) .
$$

Let $C$ be a subset of $M$ (i.e. the union of all $\mathbf{D}(M), \mathbf{D}$ a $\mathcal{L}$-sort). The language $\mathcal{L}(C)$ is the language $\mathcal{L}$ together with constant symbols for every element in $C$. Given 
an $\mathcal{L}(C)$-definable subset $X \subseteq \mathbf{D}_{x}(M)$, we say that a type $p \in S_{x}(C)$ concentrates on $X$ if $p$ contains a formula defining $X$. We denote by $S_{X}(C)$ the subset of $S_{x}(C)$ consisting of those types concentrating on $X$. For a $C$-definable function $f: X \rightarrow Y$ the pushforward of $f$ is the function $f_{*}: S_{X}(C) \rightarrow S_{Y}(C)$ sending $\operatorname{tp}(a / C)$ to $\operatorname{tp}(f(a) / C)$.

We let $\mathcal{U}$ be a monster model of $T$. As usual, a set is said to be small if it is of cardinality smaller than $|\mathcal{U}|$. A type $p(x)$ is a global type if $p \in S_{x}(\mathcal{U})$. Following Shelah's terminology, a subset $X \subseteq \mathbf{D}_{x}(M)$ is $*$-C-definable if there is a small collection $\Theta$ of $\mathcal{L}(C)$-formulas $\varphi(x)$ (where only finitely many $x_{i}$ occur in each formula) such that $X=\left\{a \in \mathbf{D}_{x}(M) \mid M \models \varphi(a), \varphi \in \Theta\right\}$.

We let dcl and acl denote the usual definable and algebraic closure modeltheoretic operators. Given a tuple of variables $x$ of length $n$, a $C$-definable set $X \subseteq \mathbf{D}_{x}(\mathcal{U})$ and a subset $A$ of $\mathcal{U}$, we let $X(A):=X \cap \operatorname{dcl}(A)^{n}$.

2.2. Definable types. We will mainly study definable types over models of $T$. Recall that given a subset $A \subseteq M$, a type $p \in S_{x}(M)$ is $A$-definable (or definable over $A$ ) if for every $\mathcal{L}$-formula $\varphi(x, y)$ there is an $\mathcal{L}(A)$-formula $d_{p}(\varphi)(y)$ such that for every $c \in \mathbf{D}_{y}(M)$

$$
\varphi(x, c) \in p(x) \Leftrightarrow M \models d_{p}(\varphi)(c) .
$$

The map $\varphi(x, y) \mapsto d_{p}(\varphi)(y)$ is called a scheme of definition for $p$, and the formula $d_{p}(\varphi)(y)$ is called a $\varphi$-definition for $p$. We say $p \in S_{x}(M)$ is definable if it is $M$ definable. Given any set $B$ containing $M$, we use $p \mid B$ to denote the type $\{\varphi(x, b) \mid$ $\left.N \models d_{p}(\varphi)(b)\right\}$, where $N$ is any model of $T$ containing $B$. We refer the reader to [18, Section 1] for proofs and details of these facts. The following is folklore.

Lemma 2.1. If $\operatorname{tp}\left(a_{1} / M\right)$ is definable and $a_{2} \in \operatorname{acl}\left(M a_{1}\right)$, then $t p\left(a_{2} / M\right)$ is definable.

Let $\varphi(x ; y)$ be a partitioned formula. A formula $\psi\left(y, z_{\varphi}\right)$ is a uniform definition for $\varphi$ (in $T$ ) if for every model $M$ of $T$ and every definable type $p \in S_{x}(M)$ there is $c=c(p, \varphi) \in \mathbf{D}_{z_{\varphi}}(M)$ such that $\psi(y, c)$ is a $\varphi$-definition for $p$. We say $T$ has uniform definability of types if every partitioned $\mathcal{L}$-formula $\varphi(x ; y)$ has a uniform definition, which we write $d(\varphi)\left(y, z_{\varphi}\right)$. The following is a routine coding exercise.

Lemma 2.2. Let $\varphi(x ; y)$ be a partitioned $\mathcal{L}$-formula. Suppose there are finitely many $\mathcal{L}$-formulas $\psi_{1}\left(y, z_{1}\right), \ldots, \psi_{n}\left(y, z_{n}\right)$ such that for every model $M$ of $T$ and every $p \in S_{x}^{\operatorname{def}}(M)$, there are $i \in\{1, \ldots, n\}$ and $c \in \mathbf{D}_{z_{i}}(M)$ such that $\psi_{i}(y, c)$ is a $\varphi$-definition for $p$. Then $\varphi(x ; y)$ has a uniform definition.

2.3. Background in valued fields. For a valued field $(K, v)$ we let $\Gamma_{K}$ denote the value group, $\mathcal{O}_{K}$ its valuation ring, $k_{K}$ the residue field and res: $\mathcal{O}_{K} \rightarrow k_{K}$ the residue map. Given a valued field extension $(K \subseteq L, v)$ and a subset $A:=$ $\left\{a_{1}, \ldots, a_{n}\right\} \subseteq L$, we say that $A$ is $K$-valuation independent if for every $K$-linear combination $\sum_{i=1}^{n} c_{i} a_{i}$ with $c_{i} \in K, v\left(\sum_{i=1}^{n} c_{i} a_{i}\right)=\min _{i}\left(v\left(c_{i} a_{i}\right)\right)$. The extension $L \mid K$ is called $v s$-defectless 1 if every finitely generated $K$-vector subspace $V$ of $L$ admits a $K$-valuation basis, that is, a $K$-valuation independent set which spans $V$ over $K$.

Let $\mathcal{L}_{\text {ring }}$ be the language of rings and $\mathcal{L}_{\text {div }}$ be its extension by a binary predicate div. We let $\mathrm{ACVF}$ be the $\mathcal{L}_{\text {div }}$-theory of algebraically closed (non-trivially) valued

\footnotetext{
${ }^{1}$ This is the same as "separated" in W. Baur's and F. Delon's terminology.
} 
fields, where div is interpreted in a valued field $(K, v)$ by $\operatorname{div}(x, y) \Leftrightarrow v(x) \leqslant v(y)$. Recall this theory has quantifier elimination (see also [12]).

We let $p \mathrm{CF}_{e, f}$ be the $\mathcal{L}$-theory of a finite extension $K$ of $\mathbb{Q}_{p}$ with $p$-ramification index $e$ and residue degree $f$. Here $\mathcal{L}$ is $\mathcal{L}_{\text {div }}$ together with $d=e f$ new constants symbols interpreted in any model by elements which modulo $p \mathcal{O}$ form an $\mathbb{F}_{p}$-basis of $\mathcal{O}_{K} / p \mathcal{O}_{K}$ (see [20]). Recall this theory admits quantifier elimination by adding predicates for $n^{\text {th }}$-powers (see [20, Theorem 5.6]).

Finally, the theory RCVF is the theory of real closed (non-trivially) valued fields in which the valuation ring is convex with respect to the ordering. It has quantifier elimination by results in 3 .

\section{Completions By Definable types}

3.1. The definable completion. Let $X \subseteq \mathbf{D}_{x}(\mathcal{U})$ be a $C$-definable set and $A$ be a small set containing $C$. The definable completion of $X$ over $A$, denoted $S_{X, \mathcal{U}}^{\text {def }}(A)$, is the space of $A$-definable global types which concentrate on $X$. For a tuple of variables $x$ we write $S_{x, \mathcal{U}}^{\mathrm{def}}(A)$ for $S_{\mathbf{D}_{x}(\mathcal{U}), \mathcal{U}}^{\mathrm{def}}(A)$.

Convention 3.1. In what follows, to simply notation, we will drop the index $\mathcal{U}$ in $S_{X, \mathcal{U}}^{\text {def }}(A)$ and simply write $S_{X}^{\text {def }}(A)$. Keep in mind that with this notation $S_{X}^{\text {def }}(A)$ is not a subset of $S_{X}(A)$ but a subset of $S_{X}(\mathcal{U})$. No conflict in notation will occur, as we will never consider $S_{X}^{\mathrm{def}}(A)$ as a subset of $S_{X}(A)$.

Given a $C$-definable function $f: X \rightarrow Y$, it is easy to see that the image of a definable type under $f_{*}$ is again definable. We will therefore use the more functorial notation $f^{\text {def }}: S_{X}^{\text {def }}(A) \rightarrow S_{Y}^{\text {def }}(A)$ for every small set $A$ containing $C$.

Remark 3.2.

(1) Note that if $f$ is injective, so is $f_{*}$ and hence $f^{\text {def }}$. However, the transfer of surjectivity from $f$ to $f^{\text {def }}$ is more subtle. We will say that $T$ has surjectivity transfer precisely if for every surjective definable function $f: X \rightarrow Y$, the function $f^{\text {def }}$ is surjective. Note that if $T$ has definable Skolem functions, then it has surjectivity transfer. Indeed, let $g: Y \rightarrow X$ be a definable section of $f$. Then, given any type $p \in S_{Y}^{\text {def }}$, we have that $f^{\text {def }}\left(g^{\operatorname{def}}(p)\right)=p$, so $f^{\text {def }}$ is surjective. We do not know whether CODF has surjectivity transfer.

(2) In 13, the authors considered the following variant: say $T$ has the extension property if $f^{\text {def }}$ is surjective for every surjective pro-definable map $f$ between pro-definable subsets (see Section 4.1). One can check that the latter is equivalent to surjectivity transfer. We refrain from using the expression "extension property" to avoid confusion with various occurrences of this term throughout model theory.

(3) By [13, Lemma 4.2.6], a stronger result holds for o-minimal theories and ACVF. Indeed, the function $f^{\text {def }}$ is surjective even when $X$ and $Y$ are definable subsets of products of imaginary sorts. Adapting the argument given in [13, Lemma 4.2.6(a)], the same result holds for RCVF.

\subsection{Other completions by definable types.}

3.2.1. The bounded completion. Let $T$ be an o-minimal theory and $M$ be a model of $T$. Given an elementary extension $M \preceq N$, we say that $N$ is bounded by $M$ if for every $b \in N$, there are $c_{1}, c_{2} \in M$ such that $c_{1} \leqslant b \leqslant c_{2}$. Let $A$ be a small subset of $\mathcal{U}$ and $X$ be a definable set. A type $p \in S_{X}^{\text {def }}(A)$ is bounded if for any small model 
$M$ containing $A$ and every realization $a \models p \mid M$, there is an elementary extension $M \preceq N$ with $a \in X(N)$ and such that $N$ is bounded by $M$.

Let $T$ be either RCVF or a completion of ACVF. Let $A$ be a small subset of $\mathcal{U}$. A type $p \in S_{X}^{\operatorname{def}}(A)$ is bounded if for any small model $M$ containing $A$ and every realization $a \models p \mid M, \Gamma(\operatorname{acl}(M a))$ is bounded by $\Gamma(M)$, where $\Gamma$ denotes the value group sort.

Finally, let $T$ be either an o-minimal theory, a completion of ACVF or RCVF. Let $A$ be a small subset of $\mathcal{U}$ and $X$ be a definable set. The bounded completion of $X$ over $A$, denoted $\widetilde{X}(A)$, is the set of bounded global $A$-definable types.

3.2.2. The orthogonal completion. Let $T$ be either a completion of ACVF or RCVF. Let $A$ be a small subset of $\mathcal{U}$. A type $p \in S_{x}^{\text {def }}(A)$ is said to be orthogonal to $\Gamma$ if for every model $M$ containing $A$ and every realization $a \models p \mid M, \Gamma(M)=\Gamma(\operatorname{acl}(M a))$. Given a definable set $X$, the orthogonal completion of $X$, denoted by $\widehat{X}(A)$, is the set of global $A$-definable types concentrating on $X$ which are orthogonal to $\Gamma$.

In [13, the set $\widehat{X}(A)$ is called the stable completion of $X$ over $A$. The name arises since, in this context, $\widehat{X}(A)$ also corresponds to the set of definable types over $A$ which are stably dominated, and equivalently, which are generically stable (see [13, Proposition 2.9.1]). However, such an equivalence does not hold in RCVF: every generically stable type (resp. stably dominated) must be a realized type.

\section{Remark 3.3.}

(1) If $T$ is an o-minimal expansion of the theory of real closed fields, then every bounded definable type is a realized type. However, this is not the case for general o-minimal theories. For example, the type of an element arbitrarily close to zero in DOAG is bounded.

(2) Let $T$ be either a completion of ACVF or RCVF, and let $M$ a model of $T$. If $\operatorname{tp}(a / M)$ is orthogonal to $\Gamma$, then $t p(b / M)$ is orthogonal to $\Gamma$ for every $b \in$ $\operatorname{acl}(M, a)$.

(3) Given a definable function $f: X \rightarrow Y$, then the pushforward $f_{*}$ of $f$ restricted to $\widetilde{X}$ (resp. to $\widehat{X}$ ) has image in $\widetilde{Y}$ (resp. $\widehat{Y}$ ). We use $\widetilde{f}$ (resp. $\widehat{f}$ ) to denote the restriction of $f_{*}$ to $\widetilde{X}$ (resp. $\widehat{X}$ ).

3.2.3. Geometric interpretation. For $T$ a completion of ACVF, let $V$ be a variety over a complete rank 1 valued field $F$. In [13, $\widehat{V}$ is introduced as a model-theoretic analogue of the Berkovich analytification $V^{\text {an }}$ of $V$. Similarly, our aim is to view $\widetilde{V}$ as a model-theoretic analogue of the Huber analytification of $V$. When $T$ is RCVF, $\widehat{V}$ is a good candidate to be the model-theoretic counterpart of the analytification of semi-algebraic sets defined by Jell, Scheiderer and Yu in [14]. The set $\widehat{V}$ is also tightly related to the set of residue field dominated types as defined by Ealy, Haskell and Maříková in 9 . The space $\widetilde{V}$ (in RCVF) seems to suggest there is an analogue of Huber's analytification of semi-algebraic sets. Finally, $S_{V}^{\text {def }}$ can be viewed as a model-theoretic analogue of the "space of valuations on $V$ ". As mentioned in the introduction, we will present more structural results concerning these spaces in a sequel of this article. 


\section{Spaces of Definable types as PRO-Definable Sets}

4.1. Pro-definable sets and morphisms. Let $(I, \leq)$ be a small upwards directed partially ordered set and $C$ be a small subset of $\mathcal{U}$. A $C$-definable projective system is a collection $\left(X_{i}, f_{i j}\right)$ such that:

(1) for every $i \in I, X_{i}$ is a $C$-definable set;

(2) for every $i, j \in I$ such that $i \geqslant j ; f_{i j}: X_{i} \rightarrow X_{j}$ is $C$-definable;

(3) $f_{i i}$ is the identity on $X_{i}$ and $f_{i k}=f_{j k} \circ f_{i j}$ for all $i \geqslant j \geqslant k$.

A pro-C-definable set $X$ is the projective limit $X:=\varliminf_{i \in I} X_{i}$ of a $C$-definable projective system $\left(X_{i}, f_{i j}\right)$. We say that $X$ is pro-definable if it is pro- $C$-definable for some small set of parameters $C$. Pro-definable sets can also be seen as $*$-definable sets. By a result of Kamensky [15, we may identify $X$ and $X(\mathcal{U})$.

Let $X=\lim _{i \in I} X_{i}$ and $Y=\lim _{j \in J} Y_{j}$ be two pro- $C$-definable sets with associated $C$-definable projective systems $\left(X_{i}, f_{i i^{\prime}}\right)$ and $\left(Y_{j}, g_{j j^{\prime}}\right)$. A pro-C-definable morphism is the data of a monotone function $d: J \rightarrow I$ and a family of $C$-definable functions $\left\{\varphi_{i j}: X_{i} \rightarrow Y_{j} \mid i \geqslant d(j)\right\}$ such that, for all $j \geqslant j^{\prime}$ in $J$ and all $i \geqslant i^{\prime}$ in $I$ with $i \geqslant d(j)$ and $i^{\prime} \geqslant d\left(j^{\prime}\right)$, it holds that $\varphi_{i^{\prime} j^{\prime}} \circ f_{i i^{\prime}}=g_{j j^{\prime}} \circ \varphi_{i j}$.

4.2. Completions by definable types as pro-definable sets. What does it mean that a completion by definable types is pro-definable? Let us give the precise meaning of this in the case of the definable completion. The other completions are handled analogously.

We say that definable types are pro-definable in $T$ if, for every set of parameters $C$ and for every $\mathcal{L}(C)$-definable set $X$, there is a pro- $C$-definable (possibly in $\mathcal{L}^{\text {eq }}$ ) set $P_{X}$ and a bijection $h_{X}^{M}: S_{X}^{\text {def }}(M) \rightarrow P_{X}(M)$ for every model $M$ containing $C$. Further, we require some functoriality: if $N$ is an elementary extension of $M$, we have natural maps $S_{X}^{\mathrm{def}}(M) \rightarrow S_{X}^{\mathrm{def}}(N)$ and $P_{X}(M) \rightarrow P_{X}(N)$; and if $f: X \rightarrow Y$ is an $\mathcal{L}(C)$-definable function, then there are pro- $C$-definable morphisms $f_{N}^{\prime}: P_{X}(N) \rightarrow P_{Y}(N)$ and $f_{M}^{\prime}: P_{X}(N) \rightarrow P_{Y}(N)$ making the following diagram commute

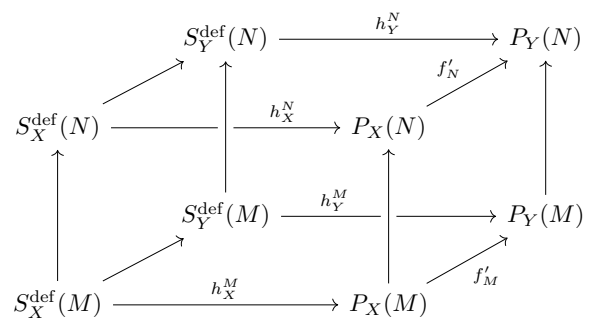

Similarly, we say a subfunctor $\mathcal{C}(\bullet)$ of $S_{\bullet}^{\text {def }}$ is pro-definable if it satisfies the same conditions after replacing $S_{\bullet}^{\text {def }}$ with $\mathcal{C}(\bullet)$ in the above diagram. It is in this sense that we say that the bounded and orthogonal completions are pro-definable (note in both cases we have a subfunctor by Remark 3.3).

The following result, essentially due to E. Hrushovski and F. Loeser [13, Lemma 2.5.1], shows the link between uniform definability of types and pro-definability. We include a proof for the reader's convenience.

Proposition 4.1. Suppose $T$ has uniform definability of types. Then definable types are pro-definable in $\mathcal{L}^{\mathrm{eq}}$. In particular, if $T$ has elimination of imaginaries, then definable types are pro-definable in $T$. 
Proof. Fix some model $M$ of $T$. Given a partitioned $\mathcal{L}$-formula $\varphi(x ; y)$, let $d(\varphi)\left(y, z_{\varphi}\right)$

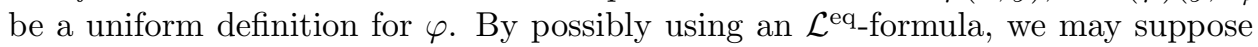
that $z_{\varphi}$ is a single variable and

$$
T^{\mathrm{eq}} \models\left(\forall z_{\varphi}\right)\left(\forall z_{\varphi}^{\prime}\right)(\forall y)\left[\left(d(\varphi)\left(y, z_{\varphi}\right) \leftrightarrow d(\varphi)\left(y, z_{\varphi}^{\prime}\right)\right) \rightarrow z_{\varphi}=z_{\varphi}^{\prime}\right] .
$$

Let $\Phi$ denote the set of partitioned $\mathcal{L}$-formulas of the form $\varphi(x ; y)$ where $y$ ranges over all finite tuples of variables. We associate to this data a map $\tau$ defined by

$$
\tau: S_{x}^{\mathrm{def}}(M) \rightarrow \prod_{\varphi \in \Phi} \mathbf{D}_{z_{\varphi}}(M) \quad p \mapsto(c(p, \varphi))_{\varphi \in \Phi},
$$

where $c(p, \varphi)$ is such that $d(\varphi)(y, c(p, \varphi))$ is a $\varphi$-definition for $p$. The codomain of $\tau$, being a small product of definable sets, is pro-definable. Thus, since $\tau$ is injective, to obtain pro-definability it suffices to show that $\tau\left(S_{x}^{\text {def }}(M)\right)$ is $*$-definable.

Without loss of generality we may suppose that the map $d$ factors through Boolean combinations, that is, $d(\varphi \wedge \psi)=d(\varphi) \wedge d(\psi)$ and $d(\neg \varphi)=\neg d(\varphi)$. Consider the following set of formulas $\Theta$ containing, for each $\mathcal{L}$-formula $\varphi(x ; y) \in \Phi$, the formula $\theta_{\varphi}\left(z_{\varphi}\right)$ given by

$$
\theta_{\varphi}\left(z_{\varphi}\right):=(\forall y)(\exists x)\left(\varphi(x, y) \leftrightarrow d(\varphi)\left(y, z_{\varphi}\right)\right) .
$$

Given $\varphi_{1}\left(x ; y_{1}\right), \ldots, \varphi_{m}\left(x ; y_{m}\right)$ in $\Phi$, let $y$ be the tuple $\left(y_{1}, \ldots, y_{m}\right)$ and $\varphi(x ; y)$ denote the conjunction $\bigwedge_{i=1}^{m} \varphi_{i}\left(x, y_{i}\right)$. Since $d$ factors through conjunctions

$$
M \models\left(\forall z_{\varphi}\right)\left(\forall z_{\varphi_{1}}\right) \cdots\left(\forall z_{\varphi_{m}}\right)(\forall y)\left(d(\varphi)\left(y, z_{\varphi}\right) \leftrightarrow \bigwedge_{i=1}^{m} d\left(\varphi_{i}\right)\left(y, z_{\varphi_{i}}\right)\right) .
$$

We claim that

$$
\tau\left(S_{X}^{\operatorname{def}}(M)\right)=\left\{\left(c_{\varphi}\right)_{\varphi} \in \prod_{\varphi \in \Phi} \mathbf{D}_{\varphi} \mid\left(c_{\varphi}\right)_{\varphi} \models \Theta\right\} .
$$

From left-to-right, let $p \in S_{x}^{\text {def }}$ and $\theta_{\varphi}\left(z_{\varphi}\right)$ be a formula in $\Theta$. We have that

$$
\tau(p) \models \theta_{\varphi}\left(z_{\varphi}\right) \Leftrightarrow \mathcal{U} \models(\forall y)(\exists x)(\varphi(x, y) \leftrightarrow d(\varphi)(y, \mathbf{c}(p, \varphi))),
$$

and the last formula holds since for every $y$ any realization of $p$ satisfies such a formula. To show the right-to-left inclusion, let $\left(c_{\varphi}\right)_{\varphi}$ be such that $\left(c_{\varphi}\right)_{\varphi} \models \Theta$. Consider the set of formulas

$$
p(x):=\left\{\varphi(x, b) \mid \mathcal{U} \models d(\varphi)\left(b, c_{\varphi}\right)\right\} .
$$

Let us show that $p(x)$ is an element of $S_{x}(\mathcal{U})$. Once we show $p(x)$ is consistent, that $p \in S_{x}^{\text {def }}(M)$ follows by definition. Let $\varphi_{1}\left(x, b_{1}\right), \ldots, \varphi_{m}\left(x, b_{m}\right)$ be formulas in $p(x)$. Letting $b:=\left(b_{1}, \ldots, b_{m}\right)$, by the definition of $p(x)$ and (E1) we have that the formula $\varphi(x, b)$ is also in $p(x)$. Moreover, since $\left(c_{\varphi}\right)_{\varphi} \models \theta_{\varphi}$ we have in particular that

$$
M \models(\exists x)\left(\varphi(x, b) \leftrightarrow d(\varphi)\left(b, c_{\varphi}\right)\right) .
$$

Finally, since $\varphi(x, b) \in p(x)$, we must have that $\models d(\varphi)\left(b, c_{\varphi}\right)$, which shows there is an element satisfying $\varphi(x, b)$. By compactness, $p(x)$ is consistent.

Given an $M$-definable subset $X \subseteq \mathbf{D}_{x}(M)$, we endow $S_{X}^{\text {def }}(M)$ with the prodefinable structure inherited from $S_{x}^{\text {def }}(M)$. More precisely, if $X$ is defined by a formula $\psi(x, a)$ for some tuple $a \in M$, we have that

$$
\tau\left(S_{X}^{\mathrm{def}}(M)\right)=\left\{\left(c_{\varphi}\right)_{\varphi} \in \prod_{\varphi \in \Phi} \mathbf{D}_{\varphi} \mid\left(c_{\varphi}\right)_{\varphi}=\Theta \cup\left\{d(\psi)\left(a, z_{\psi}\right)\right\}\right\}
$$


We leave as an exercise to show that the present construction guarantees all the above functoriality properties.

Corollary 4.2. Suppose $T$ has uniform definability of types. Let $M$ be a model and $X$ be a definable subset of $M$. Then every $*$-definable subset of $S_{X}^{\mathrm{def}}(M)$ is pro-definable.

Remark 4.3. If $T$ has uniform definability of types and has surjectivity transfer for definable functions in $\mathcal{L}^{\text {eq }}$ (see (3) of Remark 3.2), then definable types concentrating on a product of imaginary sorts are also uniformly definable. In particular, by Proposition 4.1 if $X$ is a definable subset of some product of imaginary sorts, then $S_{X}^{\text {def }}(M)$ is pro-definable (in $\mathcal{L}^{\text {eq }}$ ).

\section{StABly EMBEDDED PAIRS AND ELEMENTARITY}

Suppose $\mathcal{L}$ is a one-sorted language. Let $\mathcal{L}_{P}$ be a language extending $\mathcal{L}$ by a new unary predicate $P$. We denote an $\mathcal{L}_{P}$-structure as a pair $(N, A)$ where $N$ is an $\mathcal{L}$-structure and $A \subseteq N$ corresponds to the interpretation of $P$. Given a complete $\mathcal{L}$-theory $T$, the $\mathcal{L}_{P}$-theory of elementary pairs of models of $T$, is denoted $T_{P}$. Given a tuple $x=\left(x_{1}, \ldots, x_{m}\right)$, we abuse of notation and write $P(x)$ as an abbreviation for $\bigwedge_{i=1}^{n} P\left(x_{i}\right)$. When $\mathcal{L}$ is multi-sorted we let $\mathcal{L}_{P}$ denote the language which extends $\mathcal{L}$ by a new unary predicate $P_{\mathbf{D}}$ for every $\mathcal{L}$-sort $\mathbf{D}$. Analogously, an $\mathcal{L}_{P}$-structure $N$ is a model of $T_{P}$ if the collection of subsets $P_{\mathbf{D}}(N)$ forms an elementary $\mathcal{L}$-substructure of $N$. We will also denote any such a structure as a pair $(N, M)$ where $M \preceq N \models T$ and for every $\mathcal{L}$-sort $\mathbf{D}, P_{\mathbf{D}}(N)=\mathbf{D}(M)$.

5.1. Stable embedded pairs. Let $M \preceq N$ be an elementary extension of models of $T$. The extension is called stably embedded if for every $\mathcal{L}(N)$-definable subset $X \subseteq \mathbf{D}_{x}(N)$, the set $X \cap \mathbf{D}_{x}(M)$ is $\mathcal{L}(M)$-definable in $M$.

The class of stably embedded models of $T$ will be denoted $\mathcal{S E}(T)$. It is a standard exercise to show that $M \preceq N$ is stably embedded if and only if for every tuple $a$ in $N$, the type $t p(a / M)$ is definable.

Our main objective is to show that for various NIP theories $T, \mathcal{S E}(T)$ is an $\mathcal{L}_{P^{-}}$ elementary class. Stable theories constitute a trivial example of this phenomenon, since the class of stably embedded pairs coincides with the class of elementary pairs. O-minimal theories constitute a less trivial example. Let us first recall Marker-Steinhorn's characterizarion of definable types in o-minimal structures.

Theorem 5.1 (16, Theorem 2.1]). Let $T$ be an o-minimal theory and $M$ be a model of $T$. Then, $p \in S_{x}(M)$ is definable if and only if for every realization a of p, $M$ is Dedekind complete in $M(a)$ (where $M(a)$ is the prime model of $M$ over a).

Since the Dedekind completeness of the small structure of a pair is expressible in $\mathcal{L}_{P}$, we readily obtain:

Corollary 5.2. Let $T$ be a complete o-minimal theory. Then the class of stably embedded pairs is $\mathcal{L}_{P}$-elementary.

The following result of $\mathrm{Q}$. Brouette shows the analogue result for CODF.

Theorem 5.3 ([2, Proposition 3.6]). Let $M$ be a model of CODF. Then, $p \in S_{x}(M)$ is definable if and only if for every realization a of $p, M$ is Dedekind complete in the real closure of the ordered differential field generated by $M \cup\{a\}$. 
Corollary 5.4. The class of stably embedded pairs of models of CODF is $\mathcal{L}_{P^{-}}$ elementary.

The following characterization of definable types in $\mathbb{Z}$-groups (i.e. models of Presburger arithmetic) is due to G. Conant and S. Vojdani (see [5]).

Theorem 5.5. Let $M$ be a $\mathbb{Z}$-group. Then, $p \in S_{n}(M)$ is definable if and only if for every realization $a \models p, M \preceq M(a)$ is an end-extension.

Corollary 5.6. The class of stably embedded models of Presburger arithmetic $\mathcal{L}_{P^{-}}$ elementary.

5.2. Stable embedded pairs of valued fields. In what follows we gather the corresponding characterization of stably embedded pairs of models for ACVF, RCVF and $p \mathrm{CF}_{e, f}$. We follow the notations introduced in Section 2.3. We need first the following terminology for induced structures. Let $M$ be an $\mathcal{L}$-structure and $\mathbf{D}$ be an imaginary sort in $M^{\mathrm{eq}}$. We let $\mathcal{L}_{\mathrm{D}}$ be the language having a predicate $P_{R}$ for every $\mathcal{L}$-definable (without parameters) subset $R \subseteq \mathbf{D}^{n}(M)$. The structure $\left(\mathbf{D}(M), \mathcal{L}_{\mathbf{D}}\right)$ in which every $P_{R}$ is interpreted as the set $R$ is called the induced structure on $\mathbf{D}(M)$. The sort $\mathbf{D}$ is called stably embedded if every $\mathcal{L}^{\mathrm{eq}}(M)$-definable subset of

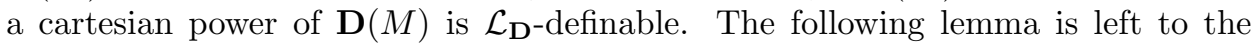
reader.

Lemma 5.7. Let $T$ be an complete $\mathcal{L}$-theory and $(N, M)$ be a stably embedded pair of models of $T$. Let $\mathbf{D}$ be an $\mathcal{L}^{\text {eq }}$-sort which is stably embedded (as a sort) in every model of $T$. Then the pair $\mathbf{D}(M) \preceq \mathbf{D}(N)$ is stably embedded in $\mathcal{L}_{\mathbf{D}}$.

Corollary 5.8. Let $T$ be RCVF, $p \mathrm{CF}_{e, f}$ or a completion of ACVF. Let $(K \preceq L, v)$ be a stably embedded pair of models of $T$. Then, the pairs $\Gamma_{K} \preceq \Gamma_{L}$ and $k_{K} \preceq k_{L}$ are stably embedded in their induced structure languages.

Proof. By Lemma 5.7, it suffices to show that in each of these theories the value group and the residue field are stably embedded (as sorts). This follows by [12, Proposition 2.1.3] for ACVF, [17, Lemma 3.13] for RCVF, and 4, Theorem 6] for $\left.p \mathrm{CF}_{e, f}\right)$.

Let us now explain how to show that the class $\mathcal{S E}(T)$ is $\mathcal{L}_{P}$-elementary for $T$ either ACVF, RCVF or $p \mathrm{CF}_{e, f}$. In all three cases, the result will follow from the following theorem:

Theorem 5.9. Let $T$ be either $\mathrm{ACVF}, \mathrm{RCVF}$ or $p \mathrm{CF}_{e, f}$. Let $K \preceq L$ be a pair of models of $T$. Then the following are equivalent

(1) the pair $K \preceq L$ is stably embedded

(2) the valued field extension $L \mid K$ is vs-defectless, the pairs $\Gamma_{K} \preceq \Gamma_{L}$ and $k_{K} \preceq k_{L}$ are stably embedded.

For ACVF the above Theorem is precisely the content of [6, Theorem 1.9]. For $\mathrm{RCVF}$ and $p \mathrm{CF}_{e, f}$ the result is new and the corresponding proof is presented in Sections 5.2 .1 and 5.2 .2 .

Corollary 5.10. Let $T$ be either $\mathrm{ACVF}, \mathrm{RCVF}$ or $p \mathrm{CF}_{e, f}$. Then the class $\mathcal{S E}(T)$ is elementary in the language of pairs.

Proof. By Theorem 5.9, it suffices to show that the condition stated in part (2) is elementary in the language of pairs. Note that being a $v s$-defectless extension 
is an elementary property in the language of pairs, so we only need to show that having stably embedded value group and residue field extensions is an elementary property in the language of pairs.

Concerning the value group, both in $\mathrm{ACVF}$ and $\mathrm{RCVF}$, the value group extension is an elementary extension of divisible ordered abelian groups and therefore, being stably embedded is elementary by Corollary 5.2 and Corollary 5.8. For $p \mathrm{CF}_{e, f}$, the value group extension is an extension of models of Presburger arithmetic, and hence the result follows by Corollary [5.6.

Regarding the residue field extension, note that it only plays a role for RCVF (indeed, in ACVF the residue field is a pure algebraically closed field, hence stable, and in $p \mathrm{CF}_{e, f}$ the residue field is finite). In $\mathrm{RCVF}$, the residue field extension corresponds to a stably embedded pair of real closed fields. As before, the result follows by Corollary 5.2 and Corollary 5.8 .

Some of these results were recently extended by P. Touchard to other classes of Henselian fields, see [22].

\subsubsection{Stably embedded pairs of real closed valued fields.}

Proof of Theorem 5.9 for RCVF. Let $(K \preceq L, v)$ be a pair of real closed valued fields.

$(1) \Rightarrow(2)$ : By Corollary [5.8, the pairs $\Gamma_{K} \preceq \Gamma_{L}$ and $k_{K} \preceq k_{L}$ are stably embedded. That the extension is $v s$-defectless follows word for word the proof for ACVF given in [6. Theorem 1.9] (it uses that the pair of value groups is a pair of pure divisible ordered abelian groups (see [17, Lemma 3.13]). Alternatively, since every model of RCVF has an elementary extension which is maximal (as a valued field), one can argue as in [22, Prop. 2.4].

$(2) \Rightarrow(1)$ : Let $X \subseteq L^{m}$ be an $\mathcal{L}_{\operatorname{div}}^{\leqslant}$-definable set over $L$. We need to show that $X \cap K^{m}$ is $\mathcal{L}_{\text {div }}^{\leqslant}$-definable over $K$. By quantifier elimination, we may suppose that $X$ is defined by one of the following formulas

(i) $v(P(x)) \square v(Q(x))$ with $\square$ either $\leqslant$ or $<$,

(ii) $0<P(x)$,

where $P, Q \in L[X]$ with $X=\left(X_{1}, \ldots, X_{m}\right)$.

Let $\left\{a_{1}, \ldots, a_{n}\right\}$ be a valuation basis for the $K$-vector space generated by the coefficients of $P$ and $Q$. For $I:=\{1, \ldots, n\}$ write $P(X)=\sum_{i \in I} a_{i} P_{i}(X)$ and $Q(X)=\sum_{i \in I} a_{i} Q_{i}(X)$, with $P_{i}, Q_{i} \in K[X]$. It then follows that for any $x \in K$ one has

$$
v(P(x))=\min _{i \in I}\left\{v\left(a_{i}\right)+v\left(P_{i}(x)\right)\right\} \text { and } v(Q(x))=\min _{i \in I}\left\{v\left(a_{i}\right)+v\left(Q_{i}(x)\right)\right\} .
$$

For (i), the result follows from (5.1) and stable embeddedness of the pair $\left(\Gamma_{L}, \Gamma_{K}\right)$ (for more details, see [6, pages 40-41]).

For (ii), multiplying the $a_{i}$ by suitable elements in $K$, we can further suppose that for every $i, j \in I$, if $v\left(a_{i}\right)$ and $v\left(a_{j}\right)$ lie in the same coset modulo $\Gamma_{K}$, then $v\left(a_{i}\right)=v\left(a_{j}\right)$. Moreover, at the expense of multiplying $P_{i}$ by -1 , we can suppose that $a_{i}>0$ for all $i \in I$.

For each $\emptyset \neq J \subseteq I$, let $A_{J}$ be the set

$$
A_{J}:=\left\{x \in K^{m} \mid v\left(\sum_{i \in I} a_{i} P_{i}(x)\right)=v\left(a_{j} P_{j}(x)\right) \text { if and only if } j \in J\right\} .
$$


By case $(i)$, we may suppose $A_{J}$ is definable over $K$. Further, since $A$ is $K$-valuation independent, the sets $A_{J}$ cover $K^{m}$ when $J$ varies over all non-empty subsets of $I$. Therefore, it suffices to show that $X \cap A_{J}$ is definable over $K$ for every $J \subseteq I$. Let us first show how to reduce to the case where $J=I$. If $J \neq I$, then for all $x \in A_{J}$ we have, since the valuation is compatible with the ordering,

$$
0<P(x) \Leftrightarrow 0<\sum_{i \in J} a_{i} P_{i}(x)+\sum_{i \in I \backslash J} a_{i} P_{i}(x) \Leftrightarrow 0<\sum_{i \in J} a_{i} P_{i}(x),
$$

and thus we obtain an equivalent formula where for all $i \in J, v\left(a_{i} P_{i}(x)\right)$ is the same. Therefore without loss of generality it suffices to show the case $J=I$. Now, since for all $x \in A_{I}, v\left(a_{i} P_{i}(x)\right)=v\left(a_{j} P_{j}(x)\right)$ for all $i, j \in I, v\left(a_{i}\right)$ and $v\left(a_{j}\right)$ are in the same coset modulo $\Gamma_{K}$, and hence $v\left(a_{i}\right)=v\left(a_{j}\right)$ for all $i, j \in I$. Also $v\left(P_{i}(x)\right)=v\left(P_{j}(x)\right)$ for all $i, j \in I$. Multiplying by a suitable constant $c \in L_{>0}$, we may suppose that $v\left(a_{i}\right)=0$ for all $i \in I$. Similarly, multiplying by a suitable constant $c^{\prime} \in K_{>0}$, we may suppose that $v\left(P_{i}(x)\right)=0$ for all $x \in A_{I}$. We conclude by noting that in this situation, for all $x \in A_{I}$

$$
0<P(x) \Leftrightarrow 0<\operatorname{res}\left(\sum_{i=1}^{n} a_{i} P_{i}(x)\right) \Leftrightarrow 0<\sum_{i=1}^{n} \operatorname{res}\left(a_{i}\right) \operatorname{res}\left(P_{i}(x)\right) .
$$

Since $k_{K}$ is stably embedded in $k_{L}$, the set $\left\{y \in k_{K}^{m} \mid 0<\sum_{i=1}^{n} \operatorname{res}\left(a_{i}\right) y_{i}\right\}$ is definable over $k_{K}$. Lifting the parameters, we obtain that $X \cap A_{I}$ is definable over $K$.

5.2.2. Stably embedded pairs of models of $p \mathrm{CF}_{e, f}$. Let $K$ be a model of $p \mathrm{CF}_{e, f}$. We need some preliminary lemmas.

Lemma 5.11. Let $(K \subseteq L, v)$ be a valued field extension. Suppose that every $y \in L$ is of the form $y=x+a$ with $a \in K$ and $x \in L$ such that $|v(x)|>\Gamma_{K}$. Then the extension is vs-defectless.

Proof. Let $V \subseteq L$ be a $K$-vector space of dimension $n$. Let us show that $V$ contains elements $\left\{x_{1}, \ldots, x_{n}\right\}$ such that all $v\left(x_{i}\right)$ lie in distinct $\Gamma_{K}$-cosets. By [10, Lemma 3.2.2], this implies that $\left\{x_{1}, \ldots, x_{n}\right\}$ is a $K$-valuation basis for $V$. We proceed by induction on $n$. Let $\left\{y_{1}, \ldots, y_{n}\right\}$ be a basis for $V$. For $n=1$ the result is trivial (take $\left.x_{1}=y_{1}\right)$. Then, by induction, the $K$-vector space generated by $\left\{y_{1}, \ldots, y_{n-1}\right\}$ contains elements $\left\{x_{1}, \ldots, x_{n-1}\right\}$ such that each $v\left(x_{i}\right)$ lies in a different $\Gamma_{K}$-coset. Without loss of generality we may assume

$$
v\left(x_{n-1}\right)+\Gamma_{K}>\cdots>v\left(x_{1}\right)+\Gamma_{K} .
$$

If either $v\left(y_{n}\right)>v\left(x_{n-1}\right)+\Gamma_{K}, v\left(x_{1}\right)>v\left(y_{n}\right)+\Gamma_{K}$, or

$$
v\left(x_{m+1}\right)>v\left(y_{n}\right)+\Gamma_{K}>v\left(x_{m}\right)+\Gamma_{K},
$$

for some $1 \leqslant m<n-1$, then we are done by setting $x_{n}:=y_{n}$. Otherwise, there are $c \in K$ and $1 \leqslant m \leqslant n-1$ such that $v\left(c y_{n}\right)=v\left(x_{m}\right)$. By assumption, there is $a \in K$ be such that $c y_{n} / x_{m}=x+a$ with $v(x)>\Gamma_{K}$. Therefore, $b:=c y_{n}-a x_{m}$ satisfies $v(b)>v\left(x_{m}\right)+\Gamma_{K}$. If $v(b)$ is in a different $\Gamma_{K}$-coset than every $v\left(x_{i}\right)$ for $i \in\{1, \ldots, n-1\}$, we are done by setting $x_{n}:=b$. Otherwise, there are $c^{\prime} \in K$ and $m^{\prime}>m$ such that $v\left(c^{\prime} b\right)=v\left(x_{m^{\prime}}\right)$. Following the same procedure, one finds a $K$-linear combination $x_{n}:=a_{n} y_{n}+\sum_{i=1}^{n-1} a_{i} x_{i}$ such that $v\left(x_{n}\right)$ is in a different $\Gamma_{K^{-}}$-coset than every $v\left(x_{i}\right)$ for $i \in\{1, \ldots, n-1\}$. 
Let $(K \subseteq L, v)$ be a valued field extension. Let $G$ be the convex hull of $\Gamma_{K}$ in $\Gamma_{L}$ and $w$ be the valuation on $L$ obtained by composing $v$ with the canonical quotient map $\Gamma_{L} \rightarrow \Gamma_{L} / G$. Let us denote $k_{K}^{w}$ and $k_{L}^{w}$ the residue fields of $(K, w)$ and $(L, w)$. As $w$ is trivial on $K, K \cong k_{K}^{w}$.

An element $a \in L$ is limit over $K$ if the extension $K(a) \mid K$ is an immediate extension. We let the reader check that if $K$ is a model of $p \mathrm{CF}_{e, f}$ and $a$ is limit over $K$, then the type $t p(a / K)$ is not definable.

Theorem 5.12 ([, Part (a) of the main Theorem]). Suppose $(K \preceq L, v)$ is a valued field extension of Henselian valued fields of characteristic 0 and let $w$ be as above. If the canonical embedding $k_{K}^{w} \rightarrow k_{L}^{w}$ is an isomorphism, then $K \preceq L$ is stably embedded in $\mathcal{L}_{\text {div }}$.

Proof of Theorem 5.9 for $p \mathrm{CF}_{e, f}$.

$(1) \Rightarrow(2)$ : That $\Gamma_{K} \preceq \Gamma_{L}$ is stably embedded follows by Corollary [5.8, It remains to show that the extension is $v s$-defectless. By Lemma 5.11, it suffices to show that every element $y \in L$ is of the form $x+a$ for $a \in K$ and $x \in L$ such that $|v(x)|>\Gamma_{K}$. Every element $y \in K$ is of this form (with $x=0$ ), so we may suppose $y \in L \backslash K$. If $|v(y)|>\Gamma_{K}$ take $a=0$. Otherwise, since by Theorem $5.5 \Gamma_{L}$ is an end extension of $\Gamma_{K}$, we must have $v(y) \in v(K)$. Suppose there is no $a \in K$ such that $|v(y-a)|>\Gamma_{K}$. Thus, for every $a \in K, v(y-a) \in \Gamma_{K}$. But this implies that $y$ is limit over $K$, which contradicts that $K$ is stably embedded in $L$. This shows the extension is $v s$-defectless.

$(2) \Rightarrow(1)$ : Since the pair is $v s$-defectless, there are no limit points in $L$ over $K$. Moreover, since $\Gamma_{K} \preceq \Gamma_{L}$ is stably embedded, by Theorem 5.5. it is an end extension of $\Gamma_{K}$. The same argument as in the previous implication shows that every element $y \in L$ is of the form $x+a$ for $a \in K$ and $x \in L$ such that $|v(x)|>\Gamma_{K}$. In particular, the convex hull of $\Gamma_{K}$ in $\Gamma_{L}$ is $\Gamma_{K}$. Let us show that $k_{L}^{w}$ is isomorphic to $K$. For all $y \in L \backslash K$ such that $w(y)=0$, there is a unique $a \in K$ such that $v(y-a)>\Gamma_{K}$. Therefore $\operatorname{res}_{w}(y)=a$, which shows that $k_{L}^{w}$ is in bijection with $K$. The result now follows from Theorem 5.12 .

We summarize the above results in the following theorem.

Theorem 5.13. Let $T$ be one of the following theories: a complete o-minimal theory; CODF; Presburger arithmetic; $p \mathrm{CF}_{e, f}$; $\mathrm{RCVF}$; a completion of ACVF. Then, the class $\mathcal{S E}(T)$ is an elementary class in $\mathcal{L}_{P}$.

Question 5.14. Is there a natural characterization of the class of complete NIP theories $T$ for which $\mathcal{S E}(T)$ is $\mathcal{L}_{P}$-elementary?

Note that there are NIP $\mathcal{L}$-theories $T$ for which the class $\mathcal{S E}(T)$ is not $\mathcal{L}_{P^{-}}$ elementary. The following example is due to L. Newelski.

Example 5.15. Let $\left(a_{i}\right)_{i<\omega}$ be an increasing sequence of irrational numbers $a_{i}$ with the limit $\lim _{i \rightarrow \infty} a_{i}=c$ irrational. Consider the structure $M=\left(\mathbb{Q},<,\left(P_{a_{i}}\right)_{i<\omega}\right)$ where $P_{a_{i}}$ is a unary predicate interpreted as the cut $\left\{x \in \mathbb{Q} \mid x<a_{i}\right\}$. The theory $T=\operatorname{Th}(M)$ is NIP as any NIP theory extended by externally definable sets is NIP (see [21, Section 3.3]). Now let $N=M \cup\left\{b_{i} \mid i<\omega\right\}$ where $b_{i}$ realizes the definable type over $M$ determined by the set of formulas

$$
\left\{c<x \mid M \models P_{a_{i}}(c)\right\} \cup\left\{x<c \mid M \models \neg P_{a_{i}}(c)\right\} .
$$


Then, $(N, M)$ is a stably embedded pair. Let $\left(N^{*}, M^{*}\right)$ be an ultrapower of $(N, M)$ over a non-principal ultrafilter $F$ over $\omega$. Let $b$ be the class of the sequence $\left(b_{i}\right)_{i<\omega}$ modulo $F$. We let the reader check that the set $X=\left\{x \in M^{*} \mid x<b\right\}$ is not definable in $M^{*}$, hence the pair $\left(N^{*}, M^{*}\right)$ is not stably embedded.

\section{UNIFORM DEFINABILITY VIA CLASSES OF PAIRS}

The following theorem provides an abstract criterion for a theory $T$ to have uniform definability of types.

Theorem 6.1. Suppose there is an $\mathcal{L}_{P}$-elementary class $\mathcal{C}$ such that

(i) if $(N, M) \in \mathcal{C}$, then $M \models T$;

(ii) if $(N, M) \in \mathcal{C}$, then $N$ is an $\mathcal{L}$-substructure of an $\mathcal{L}$-elementary extension $N^{\prime}$ of $M$;

(iii) if $(N, M) \in \mathcal{C}$, and $a$ is a finite tuple in $N$, then $t p(a / M)$ is definable (where $\operatorname{tp}(a / M)$ is defined with respect to $\left.\operatorname{Th}_{\mathcal{L}}\left(N^{\prime}\right)\right)$;

(iv) for every small model $M=T$ and every definable type $p \in S_{x}^{\mathrm{def}}(M)$, there is a pair $(N, M) \in \mathcal{C}$ and $a \in \mathbf{D}_{x}(N)$ such that $p=t p(a / M)$.

Then $T$ has uniform definability of types. In particular, definable types are prodefinable in $T$ in any reduct of $\mathcal{L}^{\mathrm{eq}}$ in which $T$ has elimination of imaginaries.

Proof. Fix a partitioned $\mathcal{L}$-formula $\varphi(x ; y)$. Let $\left(\psi_{i}\left(y, z_{i}\right)\right)_{i \in I}$ be an enumeration of all $\mathcal{L}$-formulas having $y$ among their free variables. Suppose for a contradiction that no formula $\psi_{i}\left(y, z_{i}\right)$ provides a uniform definition for $\varphi$. This implies, by Lemma 2.2, that for every finite subset $J \subseteq I$ there are a model $M_{J}$ of $T$ and a type $q_{J}(x) \in S_{x}^{\text {def }}\left(M_{J}\right)$ such that no formula $\psi_{i}$ with $i \in J$ is a $\varphi$-definition for $q_{J}$. Consider for every $i \in I$ the $\mathcal{L}_{P}$-formula $\theta_{i}(x)$

$$
\left(\forall z_{i} \in P\right)(\exists y \in P)\left(\neg\left(\varphi(x, y) \leftrightarrow \psi_{i}\left(y, z_{i}\right)\right)\right) .
$$

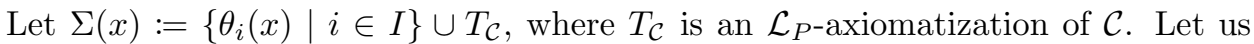
show that $\Sigma(x)$ is consistent. Let $\Sigma_{0}$ be a finite subset of $\Sigma$ and let $J:=\{i \in I \mid$ $\left.\theta_{i}(x) \in \Sigma_{0}\right\}$. By assumption, there is some $q=q_{J}(x) \in S_{x}^{\text {def }}\left(M_{J}\right)$ such that no $\psi_{i}$ with $i \in J$ is a $\varphi$-definition for $q$. By condition $(i v)$, let $\left(N, M_{J}\right)$ be an element of $\mathcal{C}$ and $a \in \mathbf{D}_{x}(N)$ be such that $q=t p(a / M)$. By the choice of $a$, we have that $\theta_{i}(a)$ holds for all $i \in J$. This shows that $\Sigma_{0}$ is consistent. Thus, by compactness, $\Sigma$ is consistent.

Let $\left(N^{\prime}, M^{\prime}\right)$ be an element in $\mathcal{C}$ and $a \in N^{\prime}$ be a realization of $\Sigma$. By the definition of $\Sigma$, the type $t p\left(a / M^{\prime}\right)$ is not definable, which contradicts $(i i i)$.

Corollary 6.2. Let $T$ be an $\mathcal{L}$-theory such that

(i) $\mathcal{S E}(T)$ is $\mathcal{L}_{P}$-elementary;

(ii) for every small model $M=T$ and every definable type $p \in S_{x}^{\mathrm{def}}(M)$, there is a stably embedded $(N, M)$ such that $p$ is realized in $N$.

Then $T$ has uniform definability of types. In particular, definable types are prodefinable in $T$ in any reduct of $\mathcal{L}^{\mathrm{eq}}$ in which $T$ has elimination of imaginaries.

Proof. This follows directly by Theorem 6.1 by taking $\mathcal{C}=\mathcal{S E}(T)$. Indeed, note that conditions $(i)-($ iii $)$ of Theorem 6.1 are trivially satisfied, and condition $(i v)$ corresponds to the present assumption $(i i)$. 
Theorem 6.3. The following theories have uniform definability of types (in one of their natural languages):

(1) any complete stable theory;

(2) any complete o-minimal theory;

(3) Presburger arithmetic;

(4) The theory of a finite extension of $\mathbb{Q}_{p}$;

(5) RCVF;

(6) any completion of ACVF;

(7) CODF.

Proof.

(1) This is a well-known result (see B. Poizat's paper [19]). Alternatively, one could also use Corollary 6.2. condition $(i)$ is trivially satisfied and for condition $(i i)$ take $N=\mathcal{U}$.

Let $T$ be one of the theories from (2)-(6). We apply Corollary 6.2. Condition $(i)$ is granted by Theorem 5.13. For condition (ii), let $M$ be a model of $T, p \in S_{x}^{\text {def }}(M)$ and $a \in \mathbf{D}_{x}(\mathcal{U})$ be a realization of $p$. If $T$ is o-minimal, by Marker-Steinhorn's theorem (Theorem 5.1), we can take $N:=M(a)$ since $(M(a), M)$ is already stably embedded. For $T$ a theory from (3)-(6) we take $N:=\operatorname{acl}(M a)$. Note that in these cases $N$ is indeed a model of $T$ and the extension $(N, M)$ is stably embedded by Lemma 2.1. This completes the result for $(2)-(6)$.

For CODF, the structure $N:=\operatorname{acl}(M a)$ is a real closed field extension of $M$ but it is not necessarily a model of CODF. To ensure condition (ii) we can apply the following idea of E. Kaplan. Let $N^{\prime}$ be a sufficiently large real closed field containing $a$ and such that the extension $\left(N^{\prime}, M\right)$ is a stably embedded extension of real closed fields. By [11, Proposition 4.11], there is a derivation $\delta$ on $N^{\prime}$ extending the derivation on $M$ such that $\left(N^{\prime}, \delta\right)$ is a model of CODF. By [2, Proposition 3.6], the extension of models of $\operatorname{CODF}\left(N^{\prime}, M\right)$ is stably embedded (in the language of CODF).

Theorem 6.4. Let $T$ be an $\mathcal{L}$-theory listed in Theorem 6.2 and $\mathcal{L}^{\prime}$ be any reduct of $\mathcal{L}^{\text {eq }}$ in which $T$ has elimination of imaginaries. Then, definable types in $T$ are pro-definable in $\mathcal{L}^{\prime}$.

Proof. This follows directly by Theorem 6.2 and Proposition 4.1

We expect similar results hold for theories of (tame) valued fields with generic derivations as defined in [7] and for theories of o-minimal fields with a generic derivation as defined in [11].

Corollary 6.5. Let $T$ be either an o-minimal theory, a completion of ACVF or $\mathrm{RCVF}$. The bounded completion of a definable set is pro-definable in $\mathcal{L}^{\mathrm{eq}}$. In the two latter cases, the orthogonal completion of a definable set is pro-definable in $\mathcal{L}^{\mathrm{eq}}$

Proof. Both cases are similar, we will just work with the bounded case and leave the other to the reader. By Corollary 4.2 it suffices to show that $\widetilde{X}(M)$ is $*$-definable inside $S_{X}^{\text {def }}(M)$, for a given $M$-definable set $X$. Suppose $X \subseteq \mathbf{D}_{x}(M)$. For every formula $\varphi(x, y, z)$ where $y$ is a $\Gamma$-variable, for every $a \in \mathbf{D}_{z}(M)$ such that $\varphi(x, y, a)$ defines a function $f_{a}: X \rightarrow \Gamma(M)$, and for every $p(x) \in \widetilde{X}(M)$, there is $\gamma \in \Gamma(M)$ such that $p(x)$ contains the formula $-\gamma<f_{a}(x)<\gamma$. Let $\psi\left(x, y^{\prime}, z\right)$ be the formula

$$
(\forall y)\left(\varphi(x, y, z) \rightarrow\left(-y^{\prime}<y<y^{\prime}\right)\right),
$$


and $d(\psi)\left(y^{\prime}, z, z_{\psi}\right)$ be its uniform definition. Let $c(p, \psi)$ be the canonical parameter such that $d(\psi)\left(y^{\prime}, z, c(p, \psi)\right)$ is the $\psi$-definition of $p(x)$. Let $\theta\left(z_{\psi}\right)$ denote the formula $(\forall z)\left(\exists y^{\prime}\right) d(\psi)\left(y^{\prime}, z, z_{\psi}\right)$. Then $p \in \widetilde{X}(M)$ if and only if for each formula $\varphi$ and associated formulas $\psi$ and $\theta$ as above

$$
M \models \theta(c(p, \psi)),
$$

which shows $\tilde{X}(M)$ is an $*$-definable subset of $S_{X}^{\text {def }}(M)$.

Question. Can one characterize NIP theories (or $d p$-minimal theories) having uniform definability of types (resp. definable types are pro-definable)?

\section{ACKNOWLEDGMEnTS}

We would like to thank: S. Starchenko and Y. Peterzil for motivating the study spaces of definable types using pairs; M. Hils for interesting discussions; L. Newelski for sharing with us Example 5.15] and E. Kaplan for pointing out a proof of Theorem 6.3 for CODF.

\section{REFERENCES}

[1] Vladimir G. Berkovich, Smooth p-adic analytic spaces are locally contractible, Invent. Math. 137 (1999), no. 1, 1-84, DOI 10.1007/s002220050323. MR.1702143

[2] Quentin Brouette, Definable types in the theory of closed ordered differential fields, Arch. Math. Logic 56 (2017), no. 1-2, 119-129, DOI 10.1007/s00153-016-0517-4. MR3598799

[3] Gregory Cherlin and Max A. Dickmann, Real closed rings. II. Model theory, Ann. Pure Appl. Logic 25 (1983), no. 3, 213-231, DOI 10.1016/0168-0072(83)90019-2. MR730855

[4] Raf Cluckers, Presburger sets and p-minimal fields, J. Symbolic Logic 68 (2003), no. 1, 153162, DOI 10.2178/jsl/1045861509. MR 1959315

[5] Gabriel Conant and Somayeh Vojdani, Definable types and f-generics in Presburger arithmetic, available at https://www.dpmms.cam.ac.uk/ gc610/Math/presburger_note.pdf.

[6] Pablo Cubides Kovacsics and Françoise Delon, Definable types in algebraically closed valued fields, MLQ Math. Log. Q. 62 (2016), no. 1-2, 35-45, DOI 10.1002/malq.201400039. MR.3472177

[7] Pablo Cubides Kovacsics and Françoise Point, Topological fields with a generic derivation, arXiv: 1912.07912 [math.LO], 2020.

[8] Françoise Delon, Définissabilité avec paramètres extérieurs dans $\mathbf{Q}_{p}$ et $\mathbf{R}$ (French, with English summary), Proc. Amer. Math. Soc. 106 (1989), no. 1, 193-198, DOI 10.2307/2047391. MR.953003

[9] Clifton Ealy, Deirdre Haskell, and Jana Maříková, Residue field domination in real closed valued fields, Notre Dame J. Form. Log. 60 (2019), no. 3, 333-351, DOI 10.1215/002945272019-0015. MR3985616

[10] Antonio J. Engler and Alexander Prestel, Valued fields, Springer Monographs in Mathematics, Springer-Verlag, Berlin, 2005. MR2183496

[11] Antongiulio Fornasiero and Elliot Kaplan, Generic derivations on o-minimal structures, arXiv:1905.07298 [math.LO], 2019.

[12] Deirdre Haskell, Ehud Hrushovski, and Dugald Macpherson, Definable sets in algebraically closed valued fields: elimination of imaginaries, J. Reine Angew. Math. 597 (2006), 175-236, DOI 10.1515/CRELLE.2006.066. MR2264318

[13] Ehud Hrushovski and François Loeser, Non-archimedean tame topology and stably dominated types, Annals of Mathematics Studies, vol. 192, Princeton University Press, Princeton, NJ, 2016. MR3445772

[14] Philipp Jell, Claus Scheiderer, and Josephine Yu, Real Tropicalization and Analytification of Semialgebraic Sets, International Mathematics Research Notices (2020), rnaa112.

[15] Moshe Kamensky, Ind- and pro- definable sets, Ann. Pure Appl. Logic 147 (2007), no. 3, 180-186, DOI 10.1016/j.apal.2007.04.003. MR2335085

[16] David Marker and Charles I. Steinhorn, Definable types in $\mathcal{O}$-minimal theories, J. Symbolic Logic 59 (1994), no. 1, 185-198, DOI 10.2307/2275260. MR.1264974 
[17] T. Mellor, Imaginaries in real closed valued fields, Ann. Pure Appl. Logic 139 (2006), no. 1-3, 230-279, DOI 10.1016/j.apal.2005.05.014. MR2206257

[18] Anand Pillay, An introduction to stability theory, Oxford Logic Guides, vol. 8, The Clarendon Press, Oxford University Press, New York, 1983. MR719195

[19] Bruno Poizat, Paires de structures stables (French), J. Symbolic Logic 48 (1983), no. 2, 239-249, DOI 10.2307/2273543. MR704080

[20] Alexander Prestel and Peter Roquette, Formally p-adic fields, Lecture Notes in Mathematics, vol. 1050, Springer-Verlag, Berlin, 1984. MR738076

[21] Pierre Simon, A guide to NIP theories, Lecture Notes in Logic, vol. 44, Association for Symbolic Logic, Chicago, IL; Cambridge Scientific Publishers, Cambridge, 2015. MR 3560428

[22] Pierre Touchard, Stably embedded submodels of henselian valued fields, arXiv preprint arXiv:2005.02363 (2020).

Mathematisches Institut der Heinrich-Heine-Universität Düsseldorf, Universitätsstr. 1, 40225 Düsseldorf, Germany

Institut de Mathématiques de Jussieu-Paris Rive Gauche, Sorbonne Université - Campus Pierre et Marie Curie 4, place Jussieu - Boite Courrier 24775252 Paris Cedex 05

Email address: jinhe.ye@imj-prg.fr 